\title{
Teste Wisconsin de Classificação de Cartas: uma revisão sistemática de 1952 a 2009
}

\author{
José Humberto da Silva-Filho' - Universidade Federal do Amazonas, Manaus, Brasil \\ Sonia Regina Pasian - Universidade de São Paulo, Ribeirão Preto, Brasil \\ Janaina Silva Martins Humberto - Universidade Federal do Amazonas, Manaus, Brasil
}

\begin{abstract}
Resumo
O WCST é um teste neuropsicológico de resolução de problemas. Os critérios de resolução são inicialmente desconhecidos e mutantes, exigindo do respondente uma compreensão dos princípios lógicos do problema. Diante da relevância do WCST na avaliação neuropsicológica, este trabalho descreve uma revisão bibliográfica dos artigos que fazem referência ao teste. Realizou-se uma busca bibliográfica em três bases internacionais: Medline, Science Direct, PsycINFO, usando como descritor o unitermo "WCST". Foram identificados 1076 artigos científicos, desde 1952 até 2009, observando-se significativo aumento das publicações com WCST a partir de 1993, com proporções crescentes a cada ano. Os países que mais publicaram sobre este teste neuropsicológico foram Holanda, Estados Unidos e Reino Unido, sendo os principais descritores das revistas que publicaram sobre o WCST: Psiquiatria, Neuropsicologia, Psicologia e Cérebro. O presente trabalho demonstra ainda que os periódicos que publicaram 10 ou mais artigos sobre o WCST têm elevado Fator de Impacto (JCR).

Palavras-chave: WCST, Wisconsin Card Sorting Test, Função Executiva, Revisão sistemática.
\end{abstract}

The Wisconsin Card Sorting Test: systematic review (1952-2009)

\begin{abstract}
The WCST is a problem-solving, neuropsychological test, with solving criteria initially unknown and mutant. Such task requires the respondent to understand the logical principles of the proposed problem. Considering the relevance of such test for neuropsychological assessment, this present study describes a bibliographic review of articles mentioning the WSCT. Such articles were searched for in three international databases (Medline, Science Direct and PsycINFO), with WSCT used as the descriptor. From 1952 to 2009, 1076 scientific articles were indentified, with a significant increase of publications from 1993 on. The countries with most studies on the WSCT were the Netherlands, USA, and UK. The main descriptors of journals that published these studies were Psychiatry, Neuropsychology, Psychology and Brain. This present study also demonstrates that the journals that published 10 or more articles on WSCT show an elevated Impact Factor (JCR).

Keywords: WCST, Wisconsin Card Sorting Test, Executive functions, Systematic review.
\end{abstract}

O Teste Wisconsin de Classificação de Cartas (Wisconsin Card Sorting Test - WCST) é um instrumento de avaliação do desempenho cognitivo. Foi originalmente desenvolvido para mensurar o raciocínio abstrato entre populações de adultos saudáveis (Berg, 1948), porém, tem sido cada vez mais empregado como um instrumento de avaliação neuropsicológica (Heaton, Chelune, Talley, Kay \& Curtiss, 1993). Sua origem se apóia em pesquisas relativas às lesões cerebrais e ao comprometimento das funções cognitivas. O WCST foi concebido para ser uma tarefa cognitiva de resolução de problemas, sendo seus critérios de resolução inicialmente desconhecidos e mutantes, exigindo do respondente uma compreensão dos princípios lógicos do problema. O seu objetivo é verificar o desempenho do indivíduo em tarefas que

\footnotetext{
${ }^{1}$ Endereço para correspondência:

Laboratório de Avaliação Psicológica - FAPSI

Universidade Federal do Amazonas

Av. General Rodrigo Octávio Jordão Ramos, 3000,

Campus Universitário, Coroado I - Manaus/Amazonas CEP:

69.000-000. Manaus/AM -

E-mail: zehumberto@uol.com.br
}

demandam as funções executivas (Heaton \& Colaboradores, 1993).

As funções executivas representam um termo genérico para um conjunto de atividades cognitivas de nível superior, imbricadas entre si e operacionalmente indissociáveis. Dizem respeito à competência cognitiva para administrar a complexidade do próprio comportamento e envolvem diversos componentes: raciocínio verbal, resolução de problemas, motivação, planejamento, flexibilidade do pensamento, memória de trabalho, formação de conceitos, abstração, tomada de decisões, juízo ético moral, execução de multitarefas, inibição de comportamentos inadequados, utilização de feedback do ambiente e habilidade para lidar com novidade (Chan, Shum, Toulopoulou, \& Chen, 2008). Tais competências têm sido associadas ao funcionamento cerebral dos lobos frontais, segundo autores clássicos como Alexander Romanovich Luria (Luria, 1966) e Muriel Deutsch Lezak (Lezak, Howieson \& Loring, 2004). As funções executivas englobam, portanto, várias habilidades cognitivas simultâneas, com finalidade de coordenar e orientar desempenhos otimizados, de forma adequada ao contexto, para alcançar objetivos futuros. Este parece 
um consenso conceitual sobre as funções executivas, segundo Rzezak e Colaboradores (2009).

O WCST é um instrumento internacionalmente reconhecido para avaliação das funções executivas, sendo frequentemente adotado em avaliações neuropsicológicas. O WCST foi considerado o mais proeminente do pequeno grupo dos chamados testes dos lobos frontais (Damásio, 2000) e internacionalmente reconhecido como padrão ouro na avaliação das funções executivas (Rzezak \& Colaboradores, 2009; Reppold, Pedrom \& Trentini, 2010). Foi desenvolvido por Esta A. Berg na Universidade de Wisconsin (EUA), em 1948, quando publicou o primeiro artigo a respeito: "A Simple Objective Technique for Measuring Flexibility in Thinking". Naquela ocasião os pesquisadores buscavam uma forma segura de medir "comportamentos abstratos" ou processos mentais envolvendo a flexibilidade de pensamento. Após este trabalho pioneiro, surgiram várias versões semelhantes do teste, baseadas na publicação original, porém com apreciáveis diferenças nos procedimentos de administração e de apuração dos resultados.

A versão do WCST mais freqüentemente referenciada na literatura internacional é a padronização de Robert K. Heaton e Colaboradores (Heaton \& Colaboradores, 1993). A padronização desenvolvida por estes autores é uma versão revisada e ampliada dos procedimentos utilizados e descritos por A. L. Robinson (Robinson \& Colaboradores, 1980) e R. K. Heaton (Heaton, 1981). Fundamentando esta nova padronização do instrumento, os autores desenvolveram um importante trabalho de revisão metodológica do WCST, descrevendo as principais diferenças entre as inúmeras versões do teste e suas variações, tanto quanto a sua administração e a atribuição de escores de desempenho.

A nova padronização do WCST propôs a unificação no manejo da técnica, tornando possíveis estudos comparativos entre resultados oriundos de diversos contextos. Esta obra (Heaton \& Colaboradores, 1993) também ofereceu novos padrões normativos do WCST, obtidos com amostra de 899 indivíduos dos EUA, de ambos os sexos, de 6,5 a 89 anos de idade, com diferentes níveis de escolaridade. Neste trabalho os pesquisadores ainda apresentaram estudos de validade e de fidedignidade. Os dados normativos foram apresentados em tabelas, distribuídos em notas Percentis, Escore T e Escore Padrão. A padronização propôs que a interpretação dos resultados fosse auxiliada por sete faixas diagnósticas, relativas ao desempenho obtido no WCST.
A literatura científica da área tem apontado estreita relação entre funções executivas e lobos préfrontais, oferecendo subsídio para que o WCST seja usualmente classificado como uma medida do funcionamento frontal. A freqüente utilização deste instrumento entre os clínicos é atribuída a sua sensibilidade com pacientes neurológicos com lesões frontais (Heaton \& Colaboradores, 1993), ou disfunções frontais.

Os argumentos acerca da distinção entre funções executivas e frontalidade também se tornaram mais robustos a partir dos dados empíricos advindos das técnicas de verificação do funcionamento cerebral em tempo real. Nesse contexto, o funcionamento executivo (também conhecido como funções cognitivas de alto nível) tem sido alvo de grande interesse das neurociências cognitivas, bem como de importantes teóricos que buscam uma integração conceitual destas funções (Tirapu-Ustárroz, MuñozCéspedes, \& Pelegrín-Valer, 2002).

A partir da padronização de Heaton (Heaton \& Colaboradores, 1993), outros pesquisadores também buscaram desenvolver novos padrões normativos locais para o WCST, em vários países e em contextos sócio-culturais específicos. Este empenho ocorreu, sobretudo, com relação às normas para crianças e adolescentes, de forma a retratar diferentes desempenhos esperados em cada faixa etária, eventuais diferenças entre sexos, em contextos sociais específicos. Em Taiwan foram desenvolvidos estudos normativos e de estrutura fatorial do WCST em adolescentes (Lin, Chen, Yang, Hsiao \& Tien, 2000). Outro estudo em Taiwan, com 219 crianças escolarizadas (idade 6-11 anos) também elaborou padrões normativos do WCST, específicos para etapas do desenvolvimento (Shu, Tien, Lung \& Chang, 2000).

Nesta mesma direção, porém avaliando adultos (idade 20-89 anos), Shan, Chen, Lee e Su (2008) examinaram 475 participantes, divididos em 10 grupos etários. Demonstraram que a faixa etária entre 20-29 anos apresentou o melhor desempenho no WCST, comparativamente aos demais indivíduos. O estudo apresentou, ainda, dados referentes ao desenvolvimento do lobo frontal, na medida em que identificou que, com o avanço da idade (após os 44 anos), há uma diminuição do volume da substância cinzenta no cérebro enquanto prossegue o aumento da substância branca, dando início aos declínios cognitivos. O estudo confirmou a existência de um declínio do desempenho no WCST no grupo com idade entre 40-49 anos. No entanto, não observou o mesmo declínio nas demais faixas etárias da amostra.

Outro trabalho nesta direção e que avaliou idosos (idade 60-80 anos) foi o desenvolvido por 
Fastenau (1998). Este pesquisador estudou a validade e as normas do WCST a partir de amostra de 63 idosos saudáveis, recorrendo a métodos de regressão para correção demográfica, apresentando resultados interessantes.

O manual do WCST de Heaton e Colaboradores (1993) foi traduzido e adaptado ao Brasil pela equipe de psicólogos coordenada pela Professora Jurema Alcides Cunha, sendo publicado pela editora Casa do Psicólogo em 2005. Esta versão brasileira do manual do WCST (Heaton, Chelune, Talley, Kay \& Curtiss, 2005) está composta por duas partes. A primeira é uma tradução autorizada do manual original integral publicado nos EUA pela Psychological Assessment Resources - PAR em 1993. A segunda parte do manual é composta por estudos brasileiros de adaptação e de validação desta técnica para nossa realidade. Jurema Alcides Cunha e colaboradores partiram de uma amostra de 515 jovens com idades entre 6,5 anos a 17 anos e 11 meses, apresentando normas avaliativas para a região da grande Porto Alegre (RS) referentes a estas idades. O estudo de validade desta adaptação brasileira foi realizado com crianças e adolescentes com diagnóstico de Transtorno do Déficit de Atenção e Hiperatividade.

Os diferentes estudos normativos com $\mathrm{O}$ WCST demonstram primeiramente a grande relevância científica desta técnica, e também o beneficio da padronização do instrumento, tornando possíveis estudos comparativos e transculturais. Em segundo lugar, devido às diferenças de desempenho no WCST encontradas em diversas culturas, observa-se a relevância da produção de padrões normativos locais, tanto de avaliação do desenvolvimento quanto intragrupos, para assegurar calibração do instrumento, visando oferecer maior precisão das medidas em avaliações neuropsicológicas.

Se parece provável que a popularidade do WCST entre clínicos e pesquisadores se deu a partir da padronização de Heaton em 1981 (Heaton \& Colaboradores, 1993), sua internacionalização e ampla difusão na comunidade científica se deram a partir da sua última padronização em 1993. Esta revisão técnica possibilitou inúmeros estudos do WCST com maior rigor na mensuração, demonstrando-se sua sensibilidade para detectar déficits em pacientes com lesões cerebrais, especialmente em lesões frontais, conforme apresentado e discutido por Rzezak e Colaboradores (2009). Neste relevante trabalho, os pesquisadores apontam as principais aplicações do WCST, bem como argumentam que as investigações com este instrumento de avaliação neuropsicológica têm obtido resultados mais estáveis na avaliação de adultos do que em crianças. Para esta faixa etária infantil faz-se necessário, segundo estes autores, reconhecer os limites técnicos do WCST, sendo recomendável a adoção de instrumentos complementares para exames mais apurados das funções executivas e do próprio funcionamento neuropsicológico global.

Diante da relevância e da visibilidade que o WCST alcançou progressivamente na literatura internacional na área da avaliação neuropsicológica, o presente trabalho teve como objetivo fazer uma revisão bibliográfica acerca deste instrumento de exame psicológico, a partir de 1952 (data da primeira publicação indexada nas bases de dados investigadas) até 2009. Buscou-se descrever os principais aspectos históricos do WCST e sua repercussão na comunidade cientifica. Por meio de procedimentos classificatórios, o presente trabalho também procurou caracterizar 1076 produções científicas relacionadas a este teste. Este material foi categorizado em função do ano e do país de publicação, descritores de áreas (subject terms) dos periódicos, além do fator de impacto dos periódicos que mais publicaram sobre o WCST.

\section{Método}

Realizou-se uma busca bibliográfica acerca do Wisconsin Card Sorting Test em três bases de dados científicas internacionais: Medline (United Satates National Library of Medicine), Science Direct (Elsevier), PsycINFO (American Psychological Association). Foi usado como descritor o termo com o qual o teste é internacionalmente reconhecido: "WCST". Foram definidos como campos de busca: título, abstract e palavras-chave. A coleta dos dados foi realizada em Setembro de 2009, sem definição de parâmetro de datas ou outros critérios de seleção dos trabalhos, visando capturar todas as publicações indexadas até o momento. Com estes parâmetros foram encontrados 708 publicações na Medline, 451 na Science Direct e 867 na PsycINFO, totalizando 2026 dados, sendo a primeira publicação indexada nestas bases de 1952. Observou-se a existência de 810 dados duplicados nas três bases, que foram removidos da amostra. Foram encontradas 1216 publicações distintas nas três bases, em três categorias: a) artigos $=1076$; b) teses $=131$; c) livros $=$ 9. O presente estudo focalizou exclusivamente os 1076 artigos científicos para comporem a amostra de pesquisas a ser analisada na presente revisão, sem critérios de exclusão de nenhum deles, por apresentar substancial representatividade dos trabalhos indexados disponíveis com o WCST.

Neste estudo foram empregados dois softwares específicos: uma ferramenta de pesquisa bibliográfica eletrônica EndNote (Thomson Reuters) e uma ferramenta 
de análise de dados estatísticos SPSS 17.0 (Statistical Package for the Social Sciences Inc). A análise dos dados foi realizada a partir de um estudo classificatório do conjunto dos abstracts encontrados a respeito do WCST, totalizando 1076. A classificação dos abstracts foi realizada a partir de quatro critérios: a) freqüência de publicações por ano; b) freqüência dos países onde os artigos foram publicados; c) freqüência dos descritores de área (subject terms) adotados pelos periódicos; d) fator de impacto dos periódicos que publicaram 10 ou mais artigos acerca do WCST, por meio do JCR (Journal Citation Reports, Science Direct), em sua última edição com base no ano de 2008.

\section{Resultados}

Freqüências de publicações por ano

Primeiramente esta amostra bibliográfica (1076 abstracts) foi analisada e classificada buscando-se identificar as datas em que elas ocorreram. O objetivo foi verificar a evolução das publicações sobre WCST ao longo do tempo, buscando depreender daí a sua repercussão na literatura internacional. Os dados demonstram que desde 1952 (quando se identificou o primeiro artigo indexado com este tema) até o ano de 1989 as publicações foram raras, representando apenas 2,69\% (29/1076) das publicações disponíveis nesta coleta. A partir dos anos 1990 as publicações cresceram significativamente. A cada intervalo de cinco anos as publicações apresentaram avanços progressivos: entre 1990-1994 identificou-se 10,25\% das publicações; entre 1995-1999, 23,42\%; entre 2000-2004, 27,41\%; e nos últimos cinco anos (2005-2009), 36,25\% dos trabalhos identificados na presente revisão bibliográfica. $\mathrm{Na}$ Figura 1 pode-se observar a profícua proliferação das publicações acerca desta técnica ao longo dos anos demonstrada pela curva ascendente, produzida a partir dos dados acumulados desde 1952 até 2009.

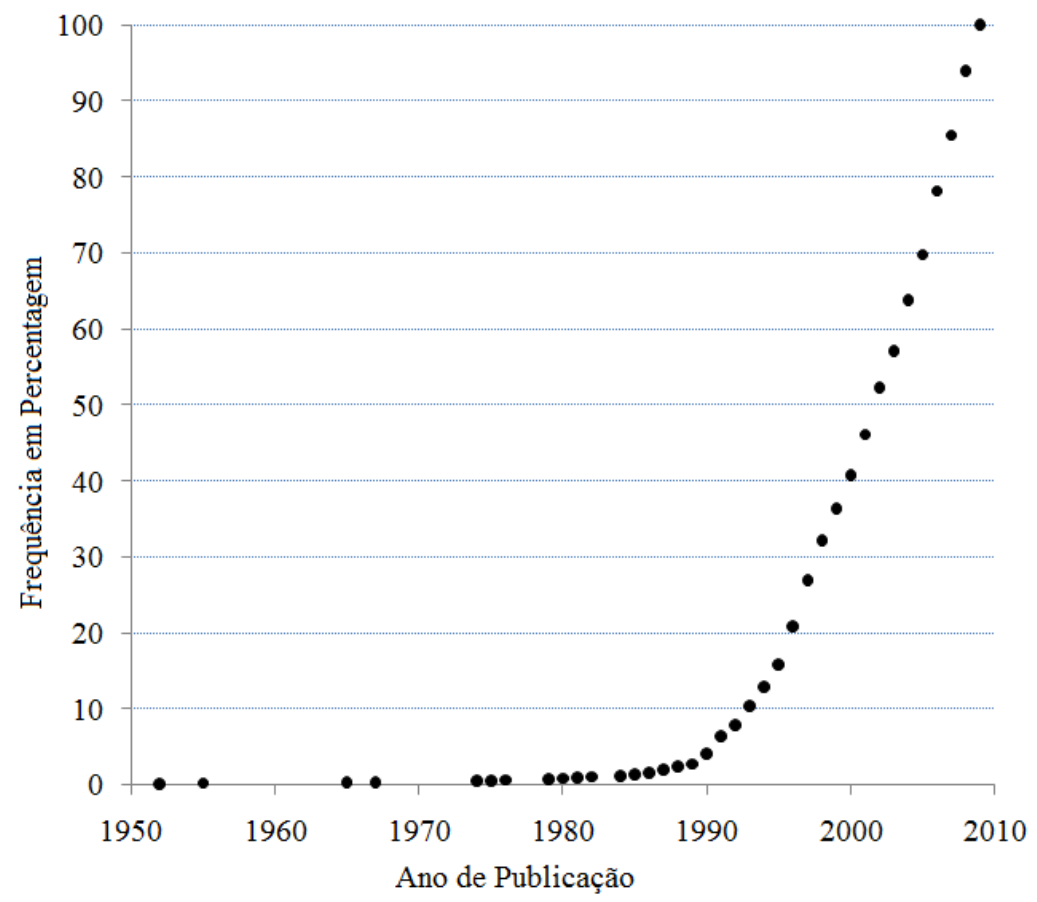

Figura 1. Distribuição de Frequência (em porcentagem) das Publicações do WCST em Função do Ano

Freqüências dos países onde os artigos foram publicados

A segunda análise deste trabalho identificou a freqüência das publicações em função dos países onde ocorreram. O objetivo foi verificar a amplitude geográfica da sua ocorrência, apesar de se levar em consideração que muitas pesquisas são publicadas em periódicos de países diferentes (Figura 2). 


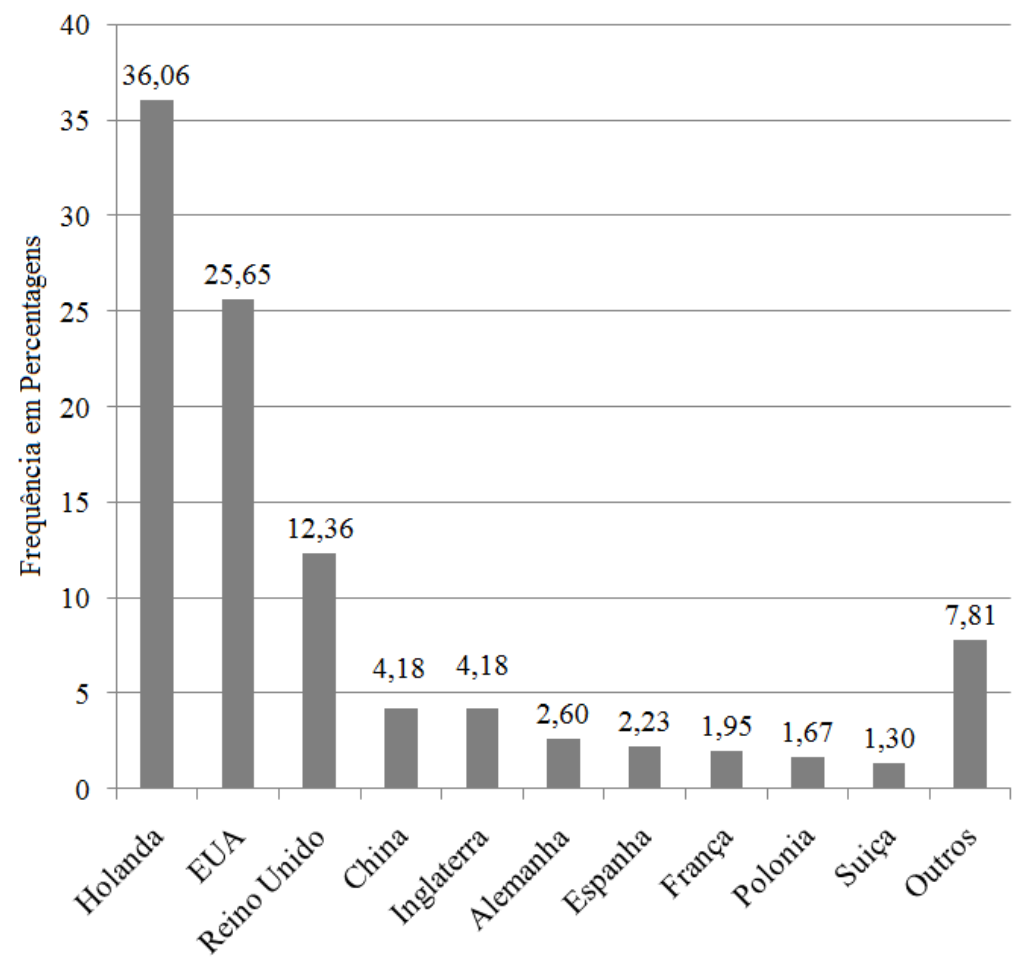

Países de Publicação

Figura 2. Distribuição de Frequência (em porcentagem) das Publicações do WCST em Função dos Países

O país que mais se destacou em publicações com o WCST foi a Holanda, com 36,06\% do total, seguido dos Estados Unidos da América, com 25,65\%. Outros países onde também houve relativa quantidade de estudos sobre este teste foram: Reino Unido $(12,36 \%)$, China (4,18\%), Inglaterra (4,18\%), Alemanha $(2,60 \%)$, Espanha (2,23\%), França (1,95\%), Polônia $(1,67 \%)$ e Suíça $(1,30 \%)$. Além desses, outros 21 países também foram identificados neste estudo. Juntos eles compõem $7,81 \%$ da amostra, com uma baixa freqüência de publicações. São eles: Japão (12 estudos), Itália (11), Turquia (11), Canadá (10), Brasil (5), Dinamarca (4), Hungria (4), Austrália (3), Áustria (3), Rússia (3), antiga Sérvia e Montenegro (3), Taiwan (3), Croácia (2), Irlanda (2), Noruega (2), Argentina (1), Coréa (1), Eslovênia (1), Índia (1), México (1) e Nova Zelândia (1).

Estes achados evidenciam que os periódicos científicos da Holanda e dos EUA atualmente lideram as publicações relacionadas ao uso do WCST. Indicam também a expansão desta técnica para outros países que começam a produzir artigos científicos sobre o tema. Apesar destes resultados já serem em parte previsíveis, visto que as bases de dados científicas aqui pesquisadas têm origem norte-americana (Medline e PsycINFO) e européia (Elsevier), os atuais resultados são ilustrativos da profusão internacional das pesquisas na área de avaliação neuropsicológica com o WCST.

Frequiência dos descritores de área (subject terms) adotados pelos periódicos

A terceira análise deste trabalho identificou a freqüência dos descritores de área (subject terms) adotados pelos periódicos onde os 1076 abstracts foram encontrados. Estes trabalhos foram publicados em um total de 331 revistas científicas, sendo que algumas delas apresentaram mais de um descritor (subject term) para demonstrar sua área de publicação. Por esta razão, o total de descritores encontrados, 471, foi superior ao número de periódicos identificados.

Os principais descritores, encontrados em 83,7\% dos periódicos foram: Psychiatry, Neurology, Psychology, Brain, Pediatrics, Psychopharmacology, Behavioral Sciences, Geriatrics, Medicine, Psychophysiology, Substance-Related Disorders, Rehabilitation, Genetics, Physiology, Neurosurgery, Neuroscience, Science. Suas freqüências de distribuição estão representadas na Figura 3, onde se identificou que os quatro primeiros descritores correspondem a $58 \%$ do total encontrado. Na categoria "outros" foram agrupados $16,1 \%$ dos descritores encontrados com baixa freqüência, sendo a maior parte deles de áreas mais específicas da saúde. 


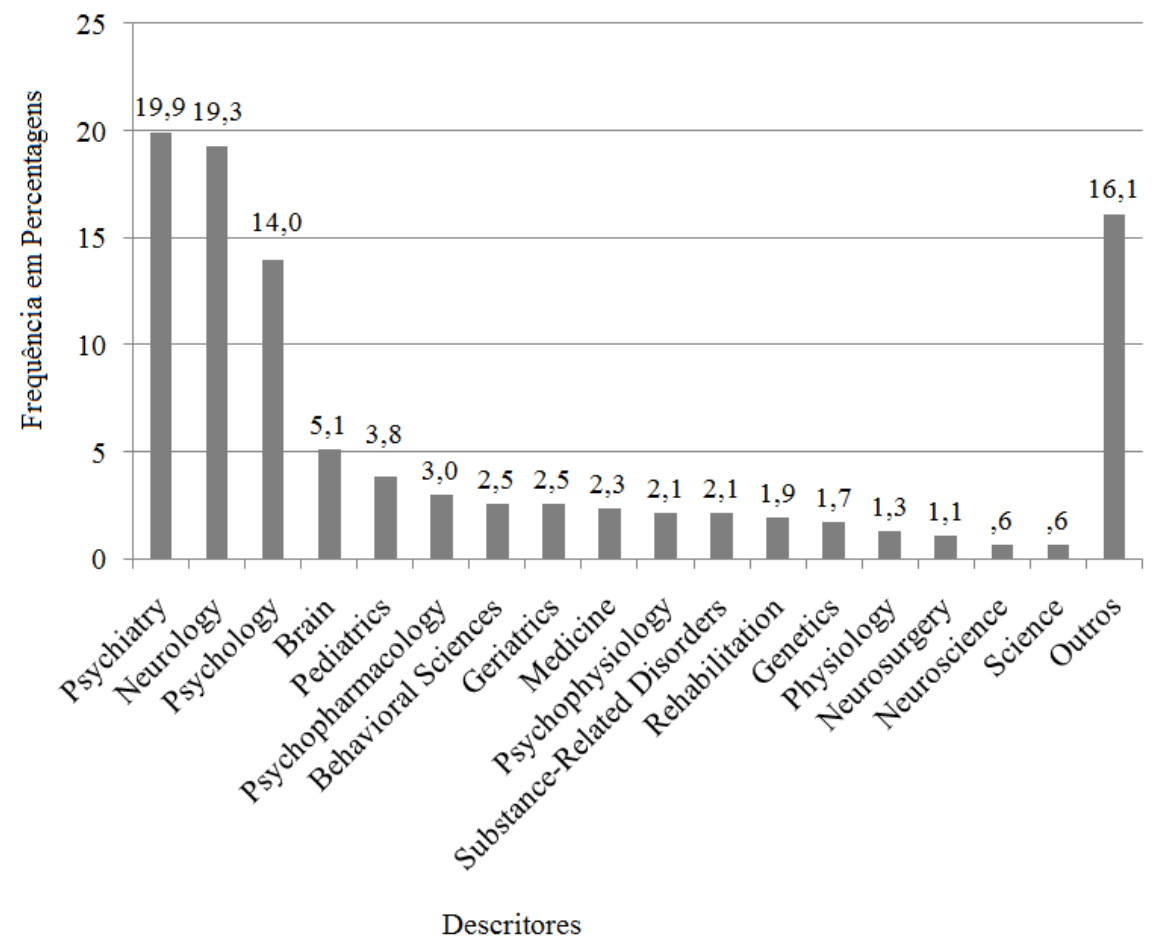

Figura 3. Distribuição de Frequência (em porcentagem) dos Descritores dos Periódicos que Publicaram Sobre o WCST

Fator de impacto dos periódicos que publicaram 10 ou mais artigos

Para esta análise, inicialmente procurou-se identificar quais das revistas científicas concentravam maior número de publicações. Os resultados demonstraram que 18 periódicos $(5,44 \%$ do total $)$ publicaram 10 ou mais artigos cada um, totalizando a proporção de $41,26 \%$ dos trabalhos identificados. Os demais 313 periódicos $(94,56 \%$ do total $)$ publicaram menos de 10 artigos sobre WCST, totalizando 58,74\% dos trabalhos aqui encontrados. Estes resultados podem ser visualizados na Figura 4.

A partir desta informação, julgou-se relevante investigar o Fator de Impacto dos periódicos que publicaram o maior volume de artigos com WCST. A busca desta informação teve como objetivo examinar o nível de reconhecimento científico destas revistas científicas, buscando uma visibilidade acerca de onde estão expostos os trabalhos com o WCST. A Tabela 1 demonstra os periódicos em ordem decrescente de freqüência (em porcentagem) em relação ao fator de impacto, acrescentando também informações sobre a quantidade de artigos, país de publicação e descritores adotados pelos periódicos. Cabe ressaltar que essa classificação do fator de impacto foi realizada apenas para os periódicos que publicaram 10 ou mais artigos acerca do WCST. Para isso, utilizou-se a referencia do Journal Citation Reports (Science Direct), em sua última edição, com base no ano de 2008.

Pode-se notar que os periódicos que mais publicaram artigos relativos ao WCST apresentam boa pontuação no fator de impacto (JCR), indicando ser uma literatura científica internacional qualificada e bem referenciada. Essa evidência pode confirmar a tendência de reconhecimento internacional da relevância do WCST em diferentes contextos de trabalho, mostrando seu rico potencial enquanto recurso técnico disponível aos profissionais da área de avaliação psicológica. 


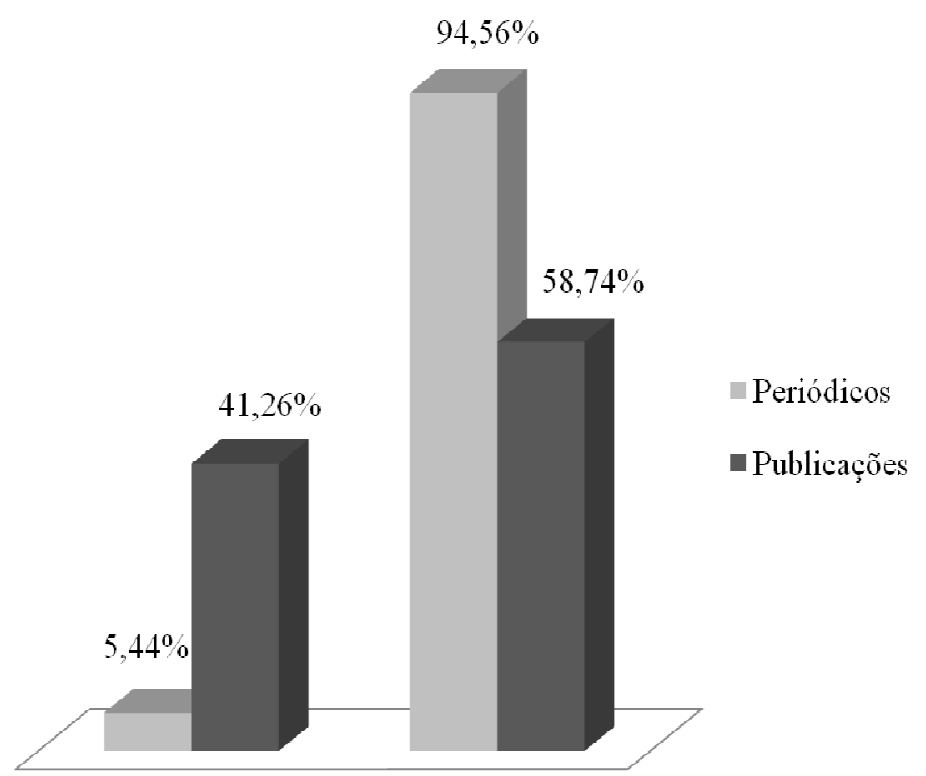

Figura 4. Distribuição das Proporções (em porcentagem) dos periódicos científicos em relação aos artigos sobre WCST neles publicados

Tabela 1 - Periódicos Com Mais Publicações Sobre WCST - Frequência, País, Descritores e Fator de Impacto (Continua)

\begin{tabular}{|c|c|c|c|c|c|}
\hline Ordem & Periódico & Frequência & País & Descritores & Fator de Impacto \\
\hline 1 & Biological Psychiatry & 39 & Holanda & $\begin{array}{c}\text { Psychiatry } \\
\text { Psychophysiology }\end{array}$ & 8.672 \\
\hline 2 & Schizophrenia Research & 102 & Holanda & Psychiatry & 4.174 \\
\hline 3 & Neuropsychologia & 20 & Holanda & Neurology & 4.074 \\
\hline 4 & $\begin{array}{l}\text { European } \\
\text { Neuropsychopharmacology }\end{array}$ & 12 & Holanda & Psychopharmacology & 3.661 \\
\hline 5 & Neuroscience & 12 & Holanda & Neurology & 3.556 \\
\hline 6 & Neuropsychology & 12 & USA & Neurology Psychology & 3.201 \\
\hline 7 & Psychiatry Research & 38 & Holanda & Psychiatry & 2.666 \\
\hline 8 & $\begin{array}{l}\text { Progress in Neuro- } \\
\text { Psychopharmacology \& } \\
\text { Biological Psychiatry }\end{array}$ & 26 & Holanda & $\begin{array}{c}\text { Psychiatry } \\
\text { Psychopharmacology }\end{array}$ & 2.638 \\
\hline 9 & $\begin{array}{l}\text { Psychiatry Research: } \\
\text { Neuroimaging }\end{array}$ & 12 & Holanda & Psychiatry & 2.638 \\
\hline 10 & $\begin{array}{l}\text { Journal of the International } \\
\text { Neuropsychological Society }\end{array}$ & 10 & USA & Neurology Psychology & 2.625 \\
\hline 11 & European Psychiatry & 17 & Holanda & Psychiatry & 2.433 \\
\hline 12 & $\begin{array}{l}\text { Journal of Clinical and } \\
\text { Experimental Neuropsychology }\end{array}$ & 20 & $\begin{array}{l}\text { Reino } \\
\text { Unido }\end{array}$ & Neurology Psychology & 2.184 \\
\hline 13 & $\begin{array}{l}\text { Archives of Clinical } \\
\text { Neuropsychology }\end{array}$ & 33 & USA & Neurology Psychology & 2.161 \\
\hline 14 & $\begin{array}{l}\text { Developmental } \\
\text { Neuropsychology }\end{array}$ & 10 & USA & Neurology Psychology & 1.964 \\
\hline
\end{tabular}


Tabela 1 - Periódicos Com Mais Publicações Sobre WCST - Frequência, País, Descritores e Fator de Impacto (Continuação)

\begin{tabular}{|c|c|c|c|c|c|}
\hline \multicolumn{2}{|r|}{ Ordem } & Periódico & \multirow{2}{*}{$\begin{array}{l}\text { Frequência } \\
\text { Reino Unido }\end{array}$} & \multirow{2}{*}{$\begin{array}{c}\text { País } \quad \text { Descritores } \\
\text { Neurology Psychology }\end{array}$} & \multirow{2}{*}{$\begin{array}{c}\text { Fator de Impacto } \\
1.750\end{array}$} \\
\hline 15 & $\begin{array}{l}\text { Clinical } \\
\text { Neuropsychologist }\end{array}$ & 31 & & & \\
\hline 16 & $\begin{array}{l}\text { Chinese Mental Health } \\
\text { Journal }\end{array}$ & 21 & China & Mental Health & Sem informação \\
\hline 17 & $\begin{array}{l}\text { Chinese Journal of Clinical } \\
\text { Psychology }\end{array}$ & 19 & China & Clinical Psychology & Sem informação \\
\hline 18 & $\begin{array}{l}\text { Am J Med Genet B } \\
\text { Neuropsychiatr Genet }\end{array}$ & 10 & USA & $\begin{array}{c}\text { Genetics, Medical } \\
\text { Neurology Psychiatry }\end{array}$ & Sem informação \\
\hline
\end{tabular}

\section{Discussão}

O WCST tem sido internacionalmente reconhecido e utilizado no campo da Psicologia, Neurologia e Psiquiatria. Tem sido um recurso sempre lembrado nas avaliações neuropsicológicas e frequentemente citado em livros didáticos sobre avaliação psicológica e correlatos. Sua aplicação objetiva oferecer auxílio técnico em processos diagnósticos de diversas condições clínicas e médicas, especialmente onde o declínio ou alterações cognitivas estejam supostamente presentes, favorecendo o trabalho multidisciplinar na área.

Os dados do presente trabalho, obtidos examinando-se 1076 abstracts publicados em 331 periódicos indexados desde 1952, demonstraram a significativa profusão de publicações científicas com o WCST, indicando que estão diretamente correlacionadas com a primeira e segunda padronização do instrumento, respectivamente em 1981 e 1993. Ou seja, num intervalo de 33 anos desde a origem do teste em 1948 até 1981, data da publicação da primeira padronização do teste (Heaton, 1981), foram identificados apenas 10 trabalhos científicos no presente estudo $(0,91 \%)$. Por outro lado, num intervalo de 12 anos, entre a primeira e a segunda padronização do WCST (1981-1993) foram identificados 101 trabalhos (9,38\%). Desde então, o que se tem observado após a publicação da segunda padronização do teste (Heaton \& Colaboradores, 1993) é uma crescente produção de trabalhos científicos. Estes dados têm demonstrado o significativo sucesso desta técnica no meio científico e, certamente, na prática clínica.

A grande abrangência geográfica das aplicações desta técnica de avaliação neuropsicológica também oferece um indicativo da sua relevância em diferentes contextos culturais. Destacaram-se as investigações científicas publicadas na Holanda (36\%), Estados Unidos (25,6\%), Reino Unido (12,4\%), China (4,2\%) e Inglaterra (4,2\%). Alemanha, Espanha, França, Polônia, Suíça estão representando juntas 9,8\% e outros 21 países representando 7,8\% das publicações. Observouse também nestes trabalhos que muitos deles buscaram produzir normas locais para o WCST.

O WCST, portanto, tem demonstrado, em ampla escala, adequados índices psicométricos, mostrando-se válido para examinar alterações nas funções executivas, levando em conta as condições socioculturais e as diferentes etapas do desenvolvimento humano. Em vista disso, tem-se observado o desenvolvimento de suas normas técnicas em diferentes países, para faixas etárias variadas, sobretudo nas idades mais suscetíveis ao efeito do desenvolvimento, como a infância (pela evolução gradual do funcionamento cognitivo) e a terceira idade (pela maior probabilidade de declínio natural), apesar de resultados menos estáveis e consistentes com as crianças, conforme apontado por Rzezak e colaboradores (2009). Ciente destas possibilidades, ao se identificar padrões de desempenho típico (normas) para determinadas faixas etárias num dado contexto sociocultural, será possível se identificar quando um indivíduo em particular se afasta da média esperada, seja para mais ou para menos, tornando então factível a formulação de hipóteses que auxiliem em processos diagnósticos de eventuais condições clínicas patológicas. Citando algumas das condições clínicas mais freqüentes onde se aplica o WCST, encontra-se na literatura científica o seu emprego para diagnóstico do Transtorno do Déficit da Atenção e Hiperatividade (TDAH), demências (sobretudo Alzheimer), esquizofrenias, traumas encefálicos, síndromes neurológicas, dentre outras. (Gioia, Isquith, Kenworthy \& Barton, 2002; Berlin, Bohlin, Nyberg \& Janols, 2004; Wagner \& Trentini, 2009).

Como referido anteriormente, os 1076 abstracts examinados no presente estudo foram publicados em 331 periódicos diferentes. Dentre eles, 18 publicaram 10 ou mais artigos sobre o WCST, correspondendo a $41,26 \%$ do total dos artigos aqui identificados, cujas publicações abrangem temas mais específicos e circunscritos da área de avaliação neuropsicológica. Por outro lado, têm-se que a maioria dos periódicos (313) 
publicaram menos de 10 artigos com o WSCT, totalizando $58,74 \%$ das investigações científicas aqui encontradas.

Examinando-se os termos descritores dos periódicos com publicações sobre WCST, observou-se que os termos "psiquiatria", "neurologia", "psicologia" e "cérebro" corresponderam juntos a $58 \%$ deles. Outros termos como pediatria, psicofarmacologia, ciências do comportamento, geriatria, medicina, psicofisiologia, desordens por abuso de substâncias, reabilitação e genética atingiram a proporção de $27 \%$ dos descritores destas revistas científicas, deixando pequena freqüência para descritores ainda mais genéricos e abrangentes dos periódicos científicos aqui examinados.

A presente revisão bibliográfica demonstrou que o WCST promoveu um significativo avanço técnico e científico na avaliação neuropsicológica internacional, sobretudo após a revisão técnica, a padronização e a atualização dos seus parâmetros normativos. Demonstrou ainda que o WCST é um instrumento mundialmente reconhecido, com estudos científicos publicados em todos os continentes, predominantemente na Europa e nos Estados Unidos. Verificou-se também a sua atualidade, pois as publicações continuam a crescer significativamente a cada ano, dando sinais de que estamos visualizando seu pleno florescer em nível internacional. Em vista disso, espera-se que o presente trabalho possa oferecer aos estudiosos desta técnica um panorama histórico da sua utilização, da sua abrangência e sua relevância enquanto instrumento de avaliação neuropsicológica.

Há ainda que se apontar neste momento que o presente trabalho, dado seu alcance informativo, tem uma contribuição circunscrita por seu próprio delineamento descritivo-histórico. Dada a abrangência temporal do presente levantamento bibliográfico e a quantidade de informações encontradas, não foi possível descrever especificamente as características dos estudos encontrados com o WCST (objetivos, amostras, resultados), marcando a limitação do presente trabalho, sobretudo pelos limites informativos dos próprios abstracts aqui examinados. Embora esta decisão técnica implique em dados de natureza geral acerca dos trabalhos encontrados, por outro lado, permitiu um mapeamento histórico relevante das investigações científicas indexadas sobre o WCST desde 1952 até 2009, sendo uma contribuição útil aos pesquisadores e aos profissionais da área de avaliação neuropsicológica.

O presente trabalho aponta para estudos futuros que podem ser desenvolvidos, priorizando-se investigações aprofundada dos próprios artigos sobre o WCST, incluindo o Fator de Impacto das próprias publicações e não somente dos periódicos científicos onde foram publicados. Estas informações poderiam contribuir para o aprimoramento técnico dos utilizadores dos instrumentos de avaliação psicológica e neuropsicológica. No entanto, espera-se que o presente trabalho possa favorecer um conhecimento histórico da expansão, repercussão e importância internacional do WCST na avaliação neuropsicológica. Espera-se estimular um uso mais adequado e embasado das próprias possibilidades interpretativas do instrumento, favorecendo a reflexão sobre o seu efetivo alcance técnico e a diversidade de aplicações em diferentes contextos.

\section{Referências}

Berg, E. A. (1948). A Simple Objective Technique for Measuring Flexibility in Thinking. The Journal of General Psychology, 39, 15-22.

Chan, R. C. K., Shum, D, Toulopoulou, T., \& Chen, E. Y. H. (2008). Assessment of executive functions: Review of instruments and identification of critical issues. Archives of Clinical Neuropsychology, 23, 201216.

Damásio, A. R. (2000). O Erro de Descartes. Emoção, Razão e Cérebro Humano. São Paulo: Companhia das Letras.

Fastenau, P. S. (1998). Validity of Regression-Based Norms: An Empirical Test of the "Comprehensive Norms" with Older Adults. Journal of Clinical and Experimental Neuropsychology, 20, 906-916.

Heaton, R. K., Chelune, G. J., Talley, J. L., Kay, G. G., \& Curtiss, G. (1993). Wisconsin Card Sorting Test Manual (Revised and Expanded). Odessa, Psychological Assessment Resources - PAR.

Heaton, R. K., Chelune, G. J., Talley, J. L., Kay, G. G., \& Curtiss, G. (2005). Manual do Teste Wisconsin de Classificação de Cartas. São Paulo: Editora Casa do Psicólogo.

Heaton, R. K. (1981). A manual for the Wisconsin Card Sorting Test. Odessa, Psychological Assessment Resources - PAR.

Lezak, M. D., Howieson, D. B., \& Loring, D. W. (2004). Neuropsychological Assessment. Oxford University Press.

Lin, C. C. H., Chen, W. J., Yang, H., Hsiao, C. K., \& Tien, A. Y. (2000). Performance on the Wisconsin Card Sorting Test among adolescents in Taiwan: Norms, factorial structure, and relation to schizotypy. Journal of Clinical and Experimental Neuropsychology, 22(1), 69-79

Luria, A. R. (1966). Higer Cortical Functions in man. London, Tavistock Publications. 
Robinson, A.L., Heaton, R. K., Lehman, R. A., \& Stilson, D. W. (1980) The utility of the Wisconsin Card Sorting Test in detecting and localizing frontal brain lesions. Journal of Consulting and Clinical Psychology, 48, 605-614.

Reppold, C. T., Pedrom, A. C., \& Trentini, C. M. (2010). Avaliação das funções executivas por meio do Teste Wisconsin de Classificação de Cartas versão computadorizada. Em M. C. R. A. Joly, \& C. T. Reppold, (Orgs). Estudos de testes informatizados para avaliação psicológica (pp 45-62). São Paulo. Capsi Livraria e Editora Ltda.

Rzezak, P., Fuentes, D., Guimarães, C. A., ThomeSouza, S., Kuczynski, E. Guerreiro, M., \& Valente, K. D. R. (2009). Executive dysfunction in children and adolescents with temporal lobe epilepsy: Is the Wisconsin Card Sorting Test enough? Epilepsy and Behavior, 15, 376-381.
Shan, I. K., Chen, Y. S., Lee, Y. C., \& Su, T. P. (2008). Adult Normative Data of the Wisconsin Card Sorting Test in Taiwan. Journal of the Chinese Medical Association, 71(10), 517-522.

Shu, B. C., Tien, A. Y., Lung, F. W., \& Chang, Y. Y. (2000). Norms for the Wisconsin Card Sorting Test in 6- to 11-year-old children in Taiwan. Clinical Neuropsychologist, 14(3), 275-286.

Tirapu-Ustárroz, J., Muñoz-Céspedes, J. M., \& Pelegrín-Valer, C. (2002). Funciones ejecutivas: necesidad de uma integración conceptual. Revista de Neurologia, 34(7), 673-685.

Recebido em setembro de 2010

Reformulado em janeiro de 2011

Aprovado em março de 2011

Sobre os autores:

José Humberto da Silva-Filho é Psicológo. Especialista em psicologia Clinica. Especialista em Neuropsicologia. Doutor em Psicologia (FFCLRP-USP). Atualmente é docente (graduação e pós-graduação) da Faculdade de Psicologia da Universidade Federal do Amazonas (UFAM). Fundou e Coordena o Laboratório de Avaliação Psicológica do Amazonas. Tem experiência na área de Psicologia, com ênfase em construção e validade de testes, escalas e outras medidas psicológicas, atuando principalmente nas áreas de psicologia cognitiva e neuropsicologia.

Sonia Regina Pasian é Psicóloga, Mestre em Epistemologia da Psicologia e da Psicanálise (UFSCar), Doutora em Saúde Mental (FMRP - USP), atualmente docente (graduação e pós-graduação) do Curso de Psicologia do Departamento de Psicologia e Educação da Faculdade de Filosofia, Ciências e Letras de Ribeirão Preto da Universidade de São Paulo, onde coordena o Centro de Pesquisas em Psicodiagnóstico. Investiga, em especial, métodos projetivos de avaliação psicológica, focalizando processos de adaptação sócio-cultural destes instrumentos.

Janaina Silva Martins Humberto é Cirurgiã-Dentista. Mestre em Odontopediatria (UFSC). Doutora em Ciências (EERP-USP). Atualmente é docente da Faculdade de Odontologia da Universidade Federal do Amazonas (UFAM). Tem experiência na área de saúde coletiva e estudos epidemiológicos. 